Ramy El Khoury, MD

Richard Jung, MD

Ashish Nanda, MD

Cathy Sila, MD

Michael G. Abraham,

MD

Alicia C. Castonguay, $\mathrm{PhD}$

Osama O. Zaidat, MD, MS

Correspondence \& reprint requests to Dr. Zaidat: szaidat@mcw.edu

\section{Overview of key factors in improving access to acute stroke care}

\section{ABSTRACT}

Background: Despite recent advances in acute stroke therapy, only a small proportion of patients with acute ischemic stroke receive IV and endovascular revascularization therapies. This article provides an overview of factors influencing access to stroke therapy.

Methods: The key factors influencing access to stroke care highlighted during the Society of Vascular and Interventional Neurology (SVIN) roundtable meeting are summarized. Pertinent selected references on prehospital, hospital, and legislative and economic factors influencing access to stroke care, from the Medline database (between 1995 to 2011), are included. A brief summary of these key factors in improving access to stroke therapy is provided.

Results: Prehospital factors include the community; education of hospital administrators and health care personnel; dispatchers; the medical transport system; and preparedness and stroke education of emergency medical services (EMS). Stroke-ready hospitals and networking with other regional tertiary stroke hospitals play important roles in increasing access to stroke care. In addition, legislation at the state and federal levels is a key factor in providing high-quality, timely access to stroke care for the population in general. Strategies to facilitate access to stroke therapy are critical to improving mortality and functional outcome and increasing the proportion of patients treated by systemic thrombolysis and endovascular approaches.

Conclusion: This is a brief overview and summary of selected factors influencing access to stroke care. These factors are divided into prehospital, hospital, legislative, and economic categories. Multilevel education of the population, public health care personnel, hospital preparedness, and legislative and economic factors are important in improving access to stroke care. Neurology ${ }^{\circledR}$ 2012;79 (Suppl 1):S26-S34

\section{GLOSSARY}

AIS = acute ischemic stroke; AST = acute stroke team; BAC = Brain Attack Coalition; $\mathbf{C S C}$ s = comprehensive stroke centers; EMS = emergency medical services; $\mathbf{G H}=$ general hospital; IA = intra-arterial; $\mathbf{J C}=$ Joint Commission; $\mathbf{L R}=$ likelihood ratio; NINDS = National Institute of Neurological Disorders and Stroke; OSH = outside spoke hospital; PSCs = primary stroke centers; rtPA = recombinant tissue plasminogen activator; $\mathbf{s} \mathbf{C H}=$ symptomatic intracranial hemorrhage; SLBSI = St. Luke Brain and Stroke Institute; SVIN = Society of Vascular and Interventional Neurology.

Despite approval in 1996 by the US Food and Drug Administration of IV recombinant tissue plasminogen activator (rtPA) for treatment of acute ischemic stroke (AIS) within 3 hours of symptom onset, ${ }^{1}$ only $2.4 \%$ to $5.2 \%$ of AIS patients are treated with IV rtPA. ${ }^{2-3}$ Intra-arterial (IA) thrombolytic therapy has been shown to be effective in AIS secondary to middle cerebral artery occlusions within 6 hours of symptom onset. ${ }^{4,5}$ Time is one of the most important determinants of clinical outcome in patients treated with interventional stroke therapy ${ }^{6,7}$; therefore, unless patients arrive within this narrow treatment window, even the most capable stroke centers may not have a significant clinical impact. To facilitate efficient timely access to stroke care, multidisciplinary efforts at the national level are necessary to address the main impediments to increasing access to stroke care. In 2002, the National Institute of Neurological Disorders and Stroke (NINDS) sponsored "Improving the Chain of Recovery for Acute

From the Department of Neurology (R.E.K.), University of Texas School of Medicine at Houston, Houston; Neurological Institute (R.J.), University Hospitals-Case Medical Center, Cleveland, OH; Department of Neurology (A.N.), University Hospital, University of Missouri-Columbia, Columbia; and Departments of Neurology, Neurosurgery, and Radiology (O.O.Z.), Medical College of Wisconsin/Froedtert Hospital, Milwaukee. Go to Neurology.org for full disclosures. Disclosures deemed relevant by the authors, if any, are provided at the end of this article. 
Stroke in Your Community," a symposium that analyzed 6 main barriers to stroke therapy. A panel of multidisciplinary experts proposed recommendations to overcome these barriers: public education, hospital levels of care, professional education, effective templates for stroke triage, incentives for AIS care, and provider support systems for acute stroke care. We refer the readers to the symposium proceedings at http://www.ninds.nih.gov/ news_and_events/proceedings/acute_stroke_ workshop.htm for this extensive work by the NINDS Task Force Group.

The factors influencing access to stroke therapy may also be divided into prehospital, hospital, legislative, and economic barriers (table 1). Prehospital factors include community; education of hospital administrators and health care personnel; dispatchers; medical transport systems; and preparedness and stroke education of emergency medical services (EMS). Hospital barriers may include establishing multidisciplinary protocols, triaging templates and clinical care pathways (stroke center certifications), and networking with other regional tertiary stroke hospitals. In addition, economic factors and legislation at the state and federal levels are key factors in

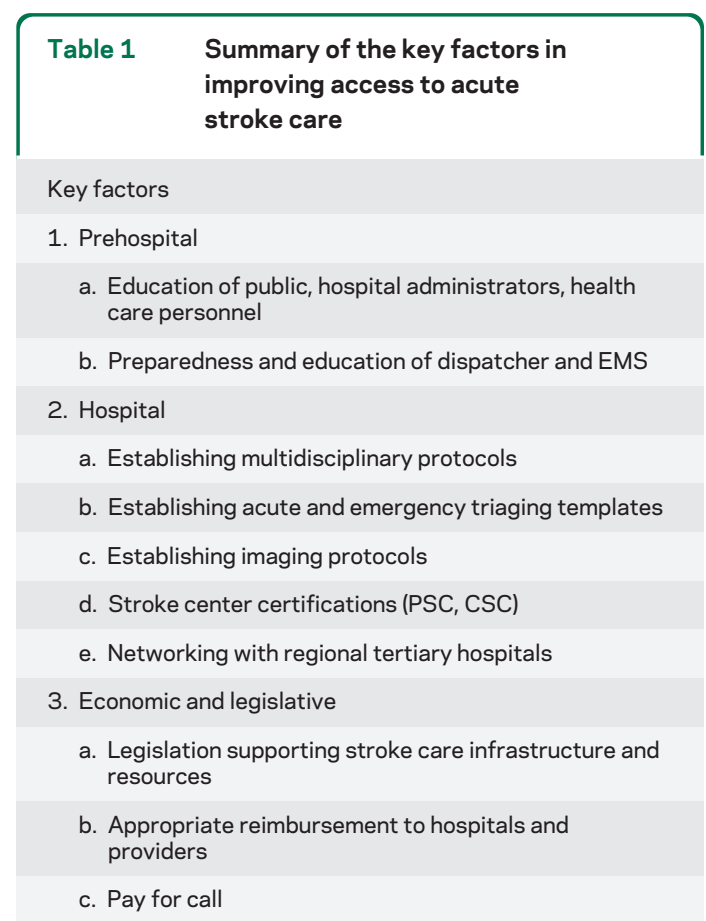

Abbreviations: $\mathrm{CSC}=$ comprehensive stroke center; $\mathrm{EMS}=$ emergency medical services; $\mathrm{PSC}=$ primary stroke center. providing timely access to high-quality stroke care for the general population.

Given the scope of this supplement and space limitations, this summary provides only a brief overview of the key factors that may influence stroke care access and is based on the presentation during the Society of Vascular and Interventional Neurology (SVIN) roundtable on endovascular AIS therapy. Some of these factors, such as stroke center certification, manpower, and training standards for interventionalists, are discussed elsewhere in this supplement.

PREHOSPITAL FACTORS Stroke education. It is imperative to provide stroke education to health care personnel, dispatchers, EMS, and the general public. Public education to identify stroke symptoms and what should be done if a stroke occurs may be accomplished via media avenues. Community outreach by members of an acute stroke team (AST) (e.g., emergency department physicians, stroke neurologists, neurointerventionalists, vascular neurosurgeons, nurses, and ancillary staff) is crucial and may be provided by hospitals, media, and public outlets. A prospective study that included a survey before and after an intense public stroke education program showed significant improvement in the public's recognition of stroke signs and symptoms after stroke education. ${ }^{8}$ Another prospective study found that participants who were exposed to a televised educational program demonstrated significantly increased knowledge of the early symptoms of stroke, compared with a control region where the program was not televised. ${ }^{9}$

Summary. The proposed recommendation is the implementation of a multilevel educational stroke program targeting the public, health care providers, dispatchers, EMS, and hospital administrators. Televised media outlets may be more effective in educating the public on stroke signs and symptoms and what to do if a stroke occurs.

Dispatchers, EMS, and patient transport. Accurate and timely identification of stroke by dispatchers and EMS providers is crucial in proceeding to the next step in the stroke chain of recovery. Education and implementation of tools for the early identification of stroke patients is a key factor to improving time from symptom onset to presentation. ${ }^{10-13}$ The use of dedicated tools and protocols was shown to improve the sensitivity and specificity of stroke diagnosis by dispatchers and EMS 
providers. ${ }^{10-13}$ Validated tools, including the Medical Priority Dispatch Systems stroke protocol, the Cincinnati Prehospital Stroke Scale (abbreviated from NIH Stroke Scale, for stroke identification in the prehospital setting), and the modified Los Angeles Prehospital Stroke Screen, enable dispatchers and EMS responders to identify stroke patients with a high degree of reliability (table 2$) \cdot{ }^{10-13}$

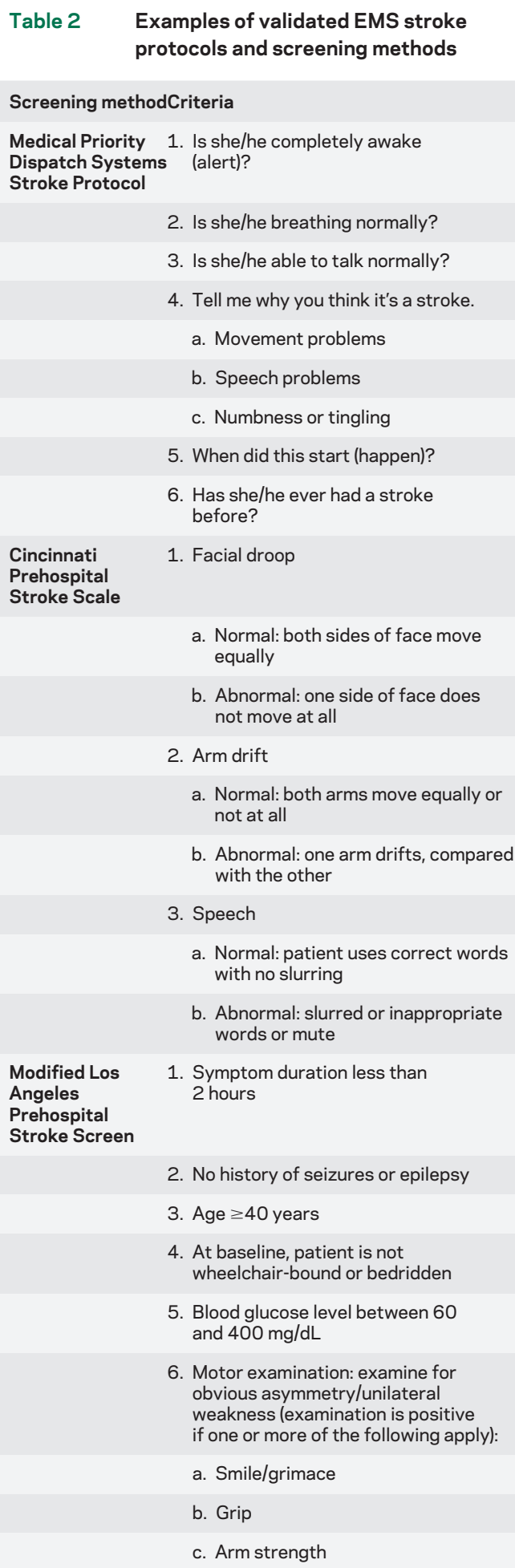

1. Symptom duration less than 2 hours

2. No history of seizures or epilepsy

3. Age $\geq 40$ years

4. At baseline, patient is not wheelchair-bound or bedridden

5. Blood glucose level between 60 and $400 \mathrm{mg} / \mathrm{dL}$

6. Motor examination: examine for obvious asymmetry/unilateral weakness (examination is positive if one or more of the following apply):

a. Smile/grimace

b. Grip

c. Arm strength

After implementation of a stroke educational program in the Houston area, the accuracy of stroke diagnosis by paramedics increased from $61 \%$ to $79 \%$, and patient admission within 2 hours of symptom onset increased from $58 \%$ to $62 \%(p=0.002) .{ }^{14}$ Similar results were noted within the metropolitan Phoenix area. Prior to the development of a prehospital EMS education program to facilitate identification and transportation of patients with AIS to predesignated primary stroke centers (PSCs), 2,947 patients were hospitalized for AIS and fewer than $1 \%$ of patients underwent thrombolysis. ${ }^{15}$ Over an 8-year period, EMS personnel were trained to identify and transport patients with AIS to PSCs, resulting in $18 \%$ of such patients undergoing thrombolysis. ${ }^{15}$

EMS transportation expedites AIS evaluation by the accepting hospital and increases the utilization rate of thrombolysis. Patients arriving via EMS are more frequently treated with IV-rtPA and have better clinical outcomes than those patients who are taken to the hospital by private car. ${ }^{2,16}$ This may be related to prearrival EMS teleconferencing with the accepting facility to increase its preparedness.

Additionally, one study showed delayed IA therapy in patients who were transferred from a community hospital to a comprehensive stroke center (CSC), vs those who had arrived directly at a CSC. Patient who arrived directly at a CSC had a shorter time to IA therapy: 234 minutes, vs 302 minutes for transferred patients. ${ }^{17}$ Another study demonstrated that transfer delays to a center performing IA therapy reduced the likelihood of emergent angiography. ${ }^{18}$ Transfer time was shown to be an independent predictor of emergent angiography (odds ratio, 0.975; 95\% confidence interval, $0.956-0.995 ; p=0.014)$; the odds of use of IA therapy decreased by $2.5 \%$ for every minute of transfer time. ${ }^{18}$

A comparison of ground vs air transport showed that $22.3 \%, 43.2 \%$, and $55.4 \%$ of Americans have access to a stroke center within 30, 45, and 60 minutes, respectively. ${ }^{19}$ The use of air ambulances would increase access to $26.0 \%, 65.5 \%$, and $79.3 \%$ within 30,45 , and 60 minutes, respectively. ${ }^{19}$ This was confirmed by another study comparing helicopter transport of 94 patients with AIS treated with IV-rtPA to 28 patients transferred by ground. Total air transport time was significantly less than ground transport time (54 vs 69 minutes; $p=0.04) \cdot{ }^{20}$ Air transport not only was more efficient but also appears to be safe. In a series of 24 stroke cases in which IV-rtPA was administered, followed by helicopter transfer, no patients had reported significant complications. ${ }^{19}$ The CSC guidelines did not specify recommended transportation time; however, they included documentation of the time between the first call of the 
transferring hospital to the CSC and the arrival time at the CSC. ${ }^{21}$

Summary. EMS transportation is associated with an increased likelihood of AIS treatment with thrombolysis. Dispatcher and EMS-validated stroke screening tools are recommended to increase the reliability of stroke diagnosis. Both air and ground transportation are considered safe methods to transfer AIS patients. Air transport should be strongly considered if endovascular therapy is a viable treatment option. Transport directly to a stroke-ready hospital (primary or comprehensive) is recommended.

HOSPITAL-RELATED FACTORS Primary stroke center designation. Establishing protocols and pathways for timely stroke care and triage may be accomplished by individual hospital effort or utilization of a state-sponsored or national certification process. In 2000, the Brain Attack Coalition (BAC) published recommendations for the establishment of primary stroke centers (PSCs), with the main goal of rapidly identifying and treating patients with AIS. ${ }^{22}$ The Joint Commission (JC) adopted these recommendations to establish PSC certification for acute stroke care hospitals. Currently, there are over 800 certified PSCs in the United States (http://www.jointcommission.org/facts_ about_primary_stroke_center_certification/).

Necessary elements of PSCs, in addition to establishing stroke care protocols and pathways, include the creation of an AST, which should consist of at least 1 physician and 1 other health care provider available 24/7. ${ }^{22}$ The AST may include members from a variety of disciplines: neurology, neurosurgery, emergency medicine, radiology, nursing, pharmacy, laboratory, physical medicine, and rehabilitation, depending on the facility's resources.

PSC designation may improve access to AIS care. One study demonstrated that in the 12 months prior to PSC certification, only 3 patients were treated with IV-rtPA, vs 44 total in the 2-year study period after certification $(p<0.0001) .{ }^{23}$ A retrospective review comparing AIS treatment in $5 \mathrm{JC}$-certified PSCs $(\mathrm{n}=302)$ and 5 non-JC-certified hospitals $(\mathrm{n}=300)$ showed that thrombolysis rates among eligible patients were significantly higher at certified PSCs than at noncertified centers $(48.2 \%$ vs $8.8 \%$; $p=0.0001) .{ }^{24}$ Furthermore, significantly more AIS patients were evaluated by a stroke team $(33 \%$ vs $0.4 \% ; p=0.0001)$ at the PSC. ${ }^{24}$ Acute neurointerventional therapy was performed in 6 patients in the PSC group, vs none at non-PSCs. ${ }^{24}$ Similar results were shown in a study of $>100,000$ stroke patients from an Illinois hospital database; IV-rtPA was administered to more patients with AIS at JC-certified PSCs than at non-JC-certified PSCs $(5.7 \%$ vs $1.2 \%$; $p<0.001) .{ }^{25}$ The New York State Stroke Center Designation Project demonstrated shorter median times from door to physician contact (10 vs $25 \mathrm{~min}$ utes) and CT performance (31 vs 40 minutes) in stroke centers than in nondesignated centers. ${ }^{26}$ In the New York Statewide Planning and Research Cooperative System database of 31,000 patients with AIS, thrombolytic therapy was utilized more frequently in stroke centers than in nondesignated centers $(4.8 \%$ vs $1.7 \% ; p<0.001) .{ }^{27}$

In the New York Statewide Planning and Research Cooperative System, admission to designated stroke centers was associated with a lower 30-day allcause mortality $(10.1 \%$ vs $12.5 \%$; $p<0.001) .{ }^{27}$ Another study, comparing 310,381 ischemic stroke discharges from JC-certified PSCs with 412,231 from noncertified hospitals, the 30-day riskstandardized mortality rate was lower in PSCs than in noncertified hospitals $(10.7 \% \pm 1.7 \%$ vs $11.0 \% \pm 1.7 \%) .^{28}$

Summary. Implementation of the components required for PSC certification appears to establish high-quality access for stroke care and increases the rate of IV-rtPA utilization.

Comprehensive stroke centers. CSCs aim to provide higher levels of stroke care with affirming availabilities of neurointerventional and neurosurgical services, establishing dedicated neurointensive care units, and offering sophisticated neuroimaging. In 2005, the BAC statement provided recommendations for the establishment of CSCs with the following components: personnel, neurointerventional and surgical therapies, neuroimaging technique, infrastructure/care setting, and educational/research programs. ${ }^{29} \mathrm{~A}$ commentary on CSC criteria is included in a separate article in this supplement (p. S239).

The interaction between CSCs and PSCs is evolving into a hub-and-spoke model with transport systems and EMS playing a vital role. The CSC serves as a resource for PSCs, offering advanced vascular neurology, neurocritical, interventional, and surgical care for patients treated initially at a PSC and providing education and expertise on managing difficult stroke cases. The CSC-PSC interlinks and their transfer protocol agreement may facilitate the development of regional stroke networks.

Currently, there are no data on the effect of CSC implementation on AIS tertiary care access in the United States. However, implementation of CSC designation in the United States is under way, which may provide future data on its effect on AIS access, outcome, and health care utilization. In Europe, which has a well-established tertiary system health 
care model, the effect of PSCs and CSCs on clinical outcome has already been evaluated. An observational study comparing AIS care in CSCs, PSCs, and general hospitals (GHs) from 1999 to 2006 in Finland demonstrated that the risk of death at 1 year was $20.8 \%$ for CSCs, $21.7 \%$ for PSCs, and $23.2 \%$ for GHs, and the utilization rate of thrombolysis was $2.2 \%$ for CSCs, vs $0.7 \%$ for PSCs, and $0.1 \%$ for GHs. ${ }^{30}$

Summary. Comprehensive stroke centers with a dedicated multidisciplinary team and sophisticated neuroimaging capabilities may provide access to high-level tertiary care services for stroke patients.

Establishing a regional network. Because of the limited number of CSCs/tertiary stroke care-ready hospitals with available infrastructure, resources, and dedicated vascular neurologists, neurointensivists, neurointerventionalists, and neurosurgeons, a regional stroke network is critical in providing timely treatment to patients with AIS. Several CSCs in the United States have established a regional stroke network system, which has improved AIS treatment and care.

One model, the Stroke Systems Initiative developed at the University Hospitals-Case Medical Center, in Cleveland, Ohio, implemented specific acute stroke clinical practice guidelines across the entire hospital system. ${ }^{31-33}$ This approach allowed for emergent reevaluation at the CSC by the AST-comprising inpatient stroke service neurology residents, vascular neurology and neurointerventional fellows and attendings, the stroke coordinator, the emergency physician, and emergency nurses - to determine the need for possible endovascular management. In order to standardize the delivery of clinical protocols, a Stroke Initiative Leadership Team, comprising neurologists, neurosurgeons, emergency physicians and nurses, neurosciences nurses, hospitalists, quality improvement professionals, and administrators from each of the system hospitals, was created to strive for consistent delivery of evidencebased care, with a quarterly review of quality assessments and measures.

This model was applied at the University Hospitals of Cleveland Medical Center, and 1 year from its inception, IV thrombolysis within the system increased from $2.4 \%$ (in 2008) to $11.5 \%$ (in 2009). Delivery of IA thrombolytic therapy increased from $25 \%$ in quarter 1 of 2008 to $43 \%$ in the first quarter of $2010 .{ }^{31}$ In summary, this system emphasizes the role of a multidisciplinary approach across all hospital systems, with shared leadership, care pathways, and protocols across emergency departments and in hospital care.

A second regional stroke network example was implemented in Kansas City, Missouri. Nurses and physicians in regional community hospital emergency departments were contacted and offered educational opportunities, both formal and informal, by the St. Luke Brain and Stroke Institute (SLBSI) physicians and nurses. Packets containing a flowsheet and all available stroke therapies were developed and distributed to community emergency departments. A primary goal of this initiative was facilitating the efficient transfer of patients. For simplicity and efficiency, a "Doctor's One Call” system was developed, in which the referring emergency department physician had to make only 1 call to a stroke nurse. After key questions were answered, a plan was made with one of the stroke neurologists, the referring physician, and the stroke nurse to proceed with the most appropriate acute stroke intervention. If endovascular treatment was planned, the neurointerventional team was ready within 45 minutes. ${ }^{32,34,35}$ A total of 248 out of 351 cases $(71 \%)$ that received AIS intervention at SLBSI were transfer cases via telephone consultation. ${ }^{35}$ This system highlights the value of providing educational modules to regional network hospitals, as well as the value of ease of access and efficient transfer of patients.

Summary. Establishing joint stroke leadership and champions from various medical specialties, stroke care stakeholders, and hospital administrators across health systems, providing resources on stroke education, and facilitating transfer to regional CSCs are important aspects of the stroke regional network of care. Regional network collaborations may be assessed by documentation of the percentage of patients with ischemic or hemorrhagic stroke or TIA transferred from another hospital to the CSC. ${ }^{21}$

TELESTROKE An important step in creating a regional stroke network is to establish methods for remote communication between different stroke system care providers and the chain of recovery. Telestroke, a branch of telemedicine, uses technology to provide the necessary communication for remote stroke care. It has 3 major advantages: providing safe acute stroke care to shortage areas, ${ }^{36-38}$ selecting patients who would potentially benefit from a higher level of care, ${ }^{39-41}$ and increasing patient enrollment in clinical trials. ${ }^{38}$

The ability to view images remotely with use of the full Digital Imaging and Communications in Medicine tool is critical for successful telestroke networking. The high-quality video teleconferencing includes communication by means of computers with webcams, smart phones, and "robots." ${ }^{2} 2$ The telestroke and robot system allows neurologists at stroke centers to evaluate patients and review imaging results via a robot that has live audio and video capabilities, allowing remote interac- 
tion between patient and doctor. A study comparing stroke telemedicine via calling with video and audio capabilities vs standard telephone-only consultation found that correct acute stroke treatment decisions were made in $98 \%$ of the telemedicine group vs $82 \%$ of the telephone-only group. ${ }^{43}$ Telestroke consultations, in comparison with telephone-only consultations, were more sensitive ( $100 \%$ vs $58 \%$ ), more specific ( $98 \%$ vs $92 \%$ ), had a more favorable positive likelihood ratio (LR; 41 vs 7) and negative likelihood ratio (LR of 0 vs 0.5 ), and had higher predictive values (positive predictive value of $94 \%$ vs $76 \%$; negative predictive value of $100 \%$ vs $84 \%$ ) for the determination of thrombolysis eligibility. ${ }^{43}$

A retrospective analysis of 353 patients with AIS in whom IV-rtPA was administered at an outside spoke hospital $(\mathrm{OSH})$ vs a hub center indicated the safety of administering thrombolysis via telestroke. ${ }^{44}$ Results showed no statistically significant differences in the rates of symptomatic intracranial hemorrhage (sICH) $(3.87 \%$ vs $5.22 \% ; p=0.58)$ and in-hospital mortality (14.92\% vs $17.39 \% ; p=0.57) .{ }^{44}$

A similar safety profile, showing no difference in the rate of sICH (6\% vs $4.5 \%$ ) or in-hospital mortality $(10.6 \%$ vs $10.7 \%)$, was found in a series of 116 patients with AIS treated with thrombolysis at the OSH vs at the hub hospital. ${ }^{45}$

In summary, immediate visual-audio interaction would increase patient access to stroke therapy and transfer for potential endovascular treatment.

HOSPITAL AND PHYSICIAN REIMBURSEMENT FOR STROKE CARE Health care for stroke patients can be costly for facilities, requiring extensive on- and off-hours resources in manpower and infrastructure to be available emergently. The lack of appropriate reimbursement to facilities and practitioners may prohibit them from providing costly AIS care. The creation of diagnosis-related group 559 (stroke patient receiving IV-rtPA) has increased hospital reimbursement to approximately US $\$ 6,000$ more per case for thrombolysis administered to patients with AIS. ${ }^{46}$ Keeping up with the constantly increasing costs for hospitals to deliver optimal stroke care, as well as incentivizing on-call stroke neurologists and telestrokologists by establishing reimbursement models for off-hours coverage and remote consultations, is an important step for payers and legislators. According to the recent Physician OnCall Pay Survey published by Sullivan, Cotter and Associates, Inc., the median hourly on-call rate paid to a Stroke Neurologist is $\$ 20.83$ for unrestricted call coverage ( $\sim 500 / 24$ hours) (http://www.sullivancotter. $\mathrm{com} /$ sullivancotter-releases-sixth-annual-physician-callpay-survey). The American Academy of Neurology
On-Call Reimbursement for Neurologists policy "believes that patients and communities will have better access to emergency neurologist services for stroke and other illnesses when neurologists are reimbursed to be on call" and that this service "should be recognized and reimbursed separately from payment for patient care" (http://www.aan.com/globals/axon/assets/2502.pdf).

Payers' support of facilities and practitioners to provide high-level AIS care on- and off-hours may translate to an initial rise in spending on stroke care. However, improved stroke systems of care may potentially result in short-term and long-term cost savings. ${ }^{47}$ In one cost-effectiveness analysis, an estimated $5 \%$ increase in IV-rtPA use nationally translated into 30,000 more patients receiving IV-rtPA annually, 4,000 patients avoiding long-term disability, and more than $\$ 100$ million per year of cost savings. ${ }^{48} \mathrm{~A}$ dedicated endovascular therapy cost-effectiveness manuscript is included in this supplement.

In summary, financial support to hospitals and providers is necessary to increase access to stroke care. Hospitals and payers need to consider pay for on-call neurologists to assess and participate in providing, supervising, or directing emergency care for stroke therapy administered locally or remotely via telestroke.

\section{LEGISLATIVE ROLE IN IMPROVING ACCESS} TO STROKE CENTERS Establishing a stroke system of care to allow timely access to high-quality stroke centers requires leadership at the local, regional, state, and national levels and support from the legislative and executive branches of the government. Efforts by stroke professional organizations and other stakeholders are also needed to ensure and support laws enacted to improve stroke care access and quality. These include The Stroke Treatment and Ongoing Prevention Stroke Act, ${ }^{49}$ a bill to "amend the Public Health Service Act to strengthen education, prevention, and treatment programs relating to stroke," and the Stroke Systems of Care Act, a national bill that requires the Department of Health and Human Services to adopt rules for coordinating stroke care services between the EMS providers and hospitals by January 1, 2014.

In the United States, legislation at the state and local levels to improve access to stroke care is well under way, as evidenced by examples such as the State of Illinois-signed Public Act 96-514 (http:// www.ilga.gov/legislation/publicacts/fulltext.asp? name $=096-0514)$ and the Los Angeles County Department of Health Services Stroke Patient Destination Policy (http://ems.dhs.lacounty.gov/policies/ Ref500/521.pdf). The Illinois state law was designed to identify hospitals capable of providing emergent stroke care and directs EMS to transport potential 
AIS patients to these hospitals, bypassing other nondesignated facilities. Similarly, the Los Angeles Department of Health policy directs the stroke patient to a certified stroke center and bypasses uncertified facilities.

A successful model to improve access to stroke therapy has been developed in other countries. The provincial government in Toronto, Canada, implemented a protocol to transport patients with AIS directly to 1 of 3 regional stroke centers, bypassing other hospitals. ${ }^{50}$ The protocol comprises a paramedic screening tool, ambulance destination decision rule, and formal memorandum of understanding by system stakeholders. The government-enforced transfer protocol resulted in a faster time of arrival to stroke centers from symptom onset and a 4-fold increase in patients who were eligible for and treated with thrombolysis, from $9.5 \%$ to $23.4 \%$ $(p=0.01) .^{50}$

In summary, legislation in support of the necessary infrastructure to increase access to stroke care and mandating EMS providers to direct stroke patients to certified centers has the potential to improve acute stroke therapy utilization.

Summary. Despite the advances made in AIS therapy, the overall treatment rate of AIS remains low. Limited access to tertiary stroke centers that provide the full spectrum of stroke treatment options may contribute to the low AIS treatment rate. Addressing key factors in the chain of recovery for stroke patients is necessary to improve access and establish an efficient stroke system of care nationwide.

Increasing public stroke awareness, educating and establishing toolkits for dispatchers and EMS providers, implementing templates and protocols for stroke centers, developing stroke center certification processes, providing telestroke capabilities, and ensuring economic and legislative support are steps needed to improve access to stroke care.

\section{AUTHOR CONTRIBUTIONS}

All authors contributed to writing and editing the final manuscript.

\section{DISCLOSURE}

Dr. El Khoury, Dr. Jung, and Dr. Nanda report no disclosures. Dr. Sila has served on the Scientific Advisory Boards for Intersocietal Accreditation Commission Board of Directors, Intersocietal Accreditation Commission for Carotid Stenting Facilities (ICACSF), and Janssen; served as Book Review Editor of Journal of Stroke and Cerebrovascular Disease; served as Stroke Section Editor for Current Cardiology Reports; has served as a consultant for Hoffman-LaRoche, Axio Research; and spouse (Gene Barnett) serves as a consultant for neurosurgical device for Elekta (Gamma Knife) and Monteris Medical (Autolitt). Dr. Abraham and Dr. Castonguay report no disclosures. Dr. Zaidat serves on the scientific advisory board for Talecris; served on the adjudication committee for Stryker; received speaker honoraria from Stryker; served on the editorial board of Frontiers in Neurology (Endovascular \& Interventional Neurology Section); serves as Editor of the Journal of Neurointerventional Surgery and serves as Associate Editor and is a member of the Editorial Board of Journal of Stroke \& Cerebrovascular Diseases; served as a consultant for Stryker
Neurovascular-Commercial, Codman Neurovascular-Commercial, and Microvention Inc, Commercial; and has received research support from Society of Vascular \& Interventional Neurology (SVIN) grant for this educational activity. Go to Neurology.org for full disclosures.

Received January 17, 2012. Accepted in final form July 2, 2012.

\section{REFERENCES}

1. The National Institute of Neurological Disorders and Stroke rt-PA Stroke Study Group. Tissue plasminogen activator for acute ischemic stroke. N Engl J Med 1995;333: 1581-1587.

2. Deng YZ, Reeves MJ, Jacobs BS, et al., Paul Coverdell National Acute Stroke Registry Michigan Prototype Investigators. IV tissue plasminogen activator use in acute stroke: experience from a statewide registry. Neurology 2006;14:306-312.

3. Adeoye O, Hornung R, Khatri P, Kleindorfer D. Recombinant tissue-type plasminogen activator use for ischemic stroke in the United States: a doubling of treatment rates over the course of 5 years. Stroke 2011;42:1952-1955.

4. Furlan A, Higashida R, Wechsler L, et al. Intra-arterial prourokinase for acute ischemic stroke: the PROACT II Study: a randomized controlled trial: Prolyse in Acute Cerebral Thromboembolism. JAMA 1999;282:2003-2011.

5. Ogawa A, Mori E, Minematsu K, et al., MELT Japan Study Group. Randomized trial of intraarterial infusion of urokinase within 6 hours of middle cerebral artery stroke: the Middle Cerebral Artery Embolism Local Fibrinolytic Intervention Trial (MELT) Japan. Stroke 2007;38:2633-2639.

6. Marler JR, Tilley BC, Lu M, et al. Early stroke treatment associated with better outcome: the NINDS rt-PA Stroke Study. Neurology 2000;55:1649-1655.

7. Khatri P, Abruzzo T, Yeatts SD, et al. Good clinical outcome after ischemic stroke with successful revascularization is time-dependent. Neurology 2009;73:1066-1072.

8. Marx JJ, Gube C, Faldum A, et al. An educational multimedia campaign improves stroke knowledge and risk perception in different stroke risk groups. Eur J Neurol 2009; 16:612-618.

9. Miyamatsu N, Kimura K, Okamura T, et al. Effects of public education by television on knowledge of early stroke symptoms among a Japanese population aged 40 to 74 years: a controlled study. Stroke 2012;43:545-549.

10. Acker JE 3rd, Pancioli AM, Crocco TJ, et al; American Heart Association; American Stroke Association Expert Panel on Emergency Medical Services Systems, Stroke Council. Implementation strategies for emergency medical services within stroke systems of care: a policy statement from the American Heart Association/American Stroke Association Expert Panel on Emergency Medical Services Systems and the Stroke Council. Stroke 2007;38:3097-3115.

11. Kothari RU, Pancioli A, Liu T, Brott T, Broderick J. Cincinnati Prehospital Stroke Scale: reproducibility and validity. Ann Emerg Med 1999;33:373-378.

12. Kidwell CS, Starkman S, Eckstein M, Weems K, Saver JL. Identifying stroke in the field: prospective validation of the Los Angeles prehospital stroke screen (LAPSS). Stroke 2000;31:71-76.

13. Buck BH, Starkman S, Eckstein M, et al. Dispatcher recognition of stroke using the National Academy Medical Priority Dispatch System. Stroke 2009;40:2027-2030.

14. Wojner-Alexandrov AW, Alexandrov AV, Rodriguez D, Persse D, Grotta JC. Houston paramedic and emergency 
stroke treatment and outcomes study (HoPSTO). Stroke 2005;36:1512-1518.

15. Demaerschalk BM, Bobrow BJ, Paulsen M. Phoenix Operation Stroke Executive Committee. Development of a metropolitan matrix of primary stroke centers: the Phoenix experience. Stroke 2008;39:1246-1253.

16. de la Ossa NP, Sánchez-Ojanguren J, Palomeras E, et al. Influence of the stroke code activation source on the outcome of acute ischemic stroke patients. Neurology 2008; 70:1238-1243.

17. Nedeltchev K, Arnold M, Brekenfeld C, et al. Pre- and in-hospital delays from stroke onset to intra-arterial thrombolysis. Stroke 2003;34:1230-1234.

18. Prabhakaran $S$, Ward E, John $S$, et al. Transfer delay is a major factor limiting the use of intra-arterial treatment in acute ischemic stroke. Stroke 2011;42:1626-1630.

18. Albright KC, Branas CC, Meyer BC, et al. ACCESS: Acute Cerebrovascular Care in Emergency Stroke Systems. Arch Neurol 2010;67:1210-1218.

19. Chalela JA, Kasner SE, Jauch EC, Pancioli AM. Safety of air medical transportation after tissue plasminogen activator administration in acute ischemic stroke. Stroke 1999; 30:2366-2368.

20. Olson MD, Rabinstein AA. Does helicopter emergency medical service transfer offer benefit to patients with stroke? Stroke 2012;43:878-880.

21. Leifer D, Bravata DM, Connors JJ 3rd, et al; American Heart Association Special Writing Group of the Stroke Council; Atherosclerotic Peripheral Vascular Disease Working Group; Council on Cardiovascular Surgery and Anesthesia; Council on Cardiovascular Nursing. Metrics for measuring quality of care in comprehensive stroke centers: detailed follow-up to Brain Attack Coalition comprehensive stroke center recommendations: a statement for healthcare professionals from the American Heart Association/American Stroke Association [erratum 2011;42: e369]. Stroke 2011;42:849-877.

22. Alberts MJ, Hademenos G, Latchaw RE, et al. Recommendations for the establishment of primary stroke centers: Brain Attack Coalition. JAMA 2000;283:3102-3109.

23. Lattimore SU, Chalela J, Davis L, et al. Impact of establishing a primary stroke center at a community hospital on the use of thrombolytic therapy: the NINDS suburban hospital stroke center experience. Stroke 2003;34:e55-e57.

24. Rajamani K, Millis S, Watson S, et al. Thrombolysis for acute ischemic stroke in joint commission-certified and -noncertified hospitals in Michigan. J Stroke Cerebrovasc Dis Epub 2011 Aug 16.

25. Prabhakaran S, McNulty M, O’Neill K, Ouyang B. Intravenous thrombolysis for stroke increases over time at primary stroke centers. Stroke 2012;43:857-877.

26. Gropen TI, Gagliano PJ, Blake CA, et al. NYSDOH Stroke Center Designation Project Workgroup. Quality improvement in acute stroke: the New York State Stroke Center Designation Project. Neurology 2006;67:88-93.

27. Xian Y, Holloway RG, Chan PS, et al. Association between stroke center hospitalization for acute ischemic stroke and mortality. JAMA 2011;305:373-380.

28. Lichtman JH, Jones SB, Wang Y, Watanabe E, LeifheitLimson E, Goldstein LB. Outcomes after ischemic stroke for hospitals with and without joint commission-certified primary stroke centers. Neurology 2011;76:1976-1982.
29. Alberts MJ, Latchaw RE, Selman WR, et al. Recommendations for comprehensive stroke centers: a consensus statement from the Brain Attack Coalition. Stroke 2005; 36:1597-1616.

30. Meretoja A, Roine RO, Kaste M, et al. Effectiveness of primary and comprehensive stroke centers: PERFECT stroke: a nationwide observational study from Finland. Stroke 2010;41:1102-1107.

31. McCartney LA, Fussner J, Richardson M, Sila C. A systems approach to stroke care: increased access to tissue plasminogen activator therapies. Stroke 2011;42:e153e154.

32. Sila C, McCartney LA, Tinsley N, et al. A systems approach to stroke care: the University Hospitals Cleveland experience. Presented at the 62nd Annual American Academy of Neurology Meeting, April 2010; Toronto.

33. Truong VV, Sundararajan S, Pundik S, DeGeorgia M, Furlan AJ, Sila C. Safety of drip and ship IV tPA in acute stroke: the University Hospitals-Case Medical Center experience. Neurology 2009;72(suppl 3):A3.

34. Rymer MM, Thrutchley DE; Stroke Team at the Mid America Brain and Stroke Institute. Organizing regional networks to increase acute stroke intervention. Neurol Res 2005;27(suppl 1):S9-S16.

35. Rymer MM. Organization of regional referral network increases the acute stroke treatment rate. Presented at the International Stroke Conference/Annual American Stroke Association Meeting; February 16-18, 2006; Kissimmee, FL.

36. Audebert HJ, Kukla C, Clarmann von Claranau S, et al. Telemedicine for safe and extended use of thrombolysis in stroke: the telemedic pilot project for integrative stroke care (TEMPiS) in Bavaria. Stroke 2005;36:287-291.

37. Meyer BC, Raman R, Hemmen T, et al. Efficacy of siteindependent telemedicine in the STRokE DOC trial: a randomised, blinded, prospective study. Lancet Neurol 2008;7:787-795.

38. Switzer JA, Hall CE, Close B, et al. A telestroke network enhances recruitment into acute stroke clinical trials. Stroke 2010;41:566-569.

39. Henninger N, Chowdhury N, Fisher M, Moonis M. Use of telemedicine to increase thrombolysis and advance care in acute ischemic stroke. Cerebrovasc Dis 2009;27(suppl 4): 9-14.

40. Silva GS, Schwamm LH. Use of telemedicine and other strategies to increase the number of patients that may be treated with intravenous thrombolysis. Curr Neurol Neurosci Rep Epub 2011 Oct 14.

41. Schwamm LH, Holloway RG, Amarenco P, et al. A review of the evidence for the use of telemedicine within stroke systems of care: a scientific statement from the American Heart Association/American Stroke Association. Stroke 2009;40:2616-2634.

42. Anderson ER, Smith B, Ido M, Frankel M. Remote assessment of stroke using the iPhone 4. J Stroke Cerebrovasc Dis Epub 2011 October 21.

43. Capampangan DJ, Wellik KE, Bobrow BJ, et al. Telemedicine versus telephone for remote emergency stroke consultations: a critically appraised topic. Neurologist 2009;15:163-166.

44. Pervez MA, Silva G, Masrur S, et al. Remote supervision of IV-tPA for acute ischemic stroke by telemedicine or telephone before transfer to a regional stroke center is feasible and safe. Stroke 2010;41:e18-e24. 
45. Martin-Schild S, Morales MM, Khaja AM, et al. Is the drip-and-ship approach to delivering thrombolysis for acute ischemic stroke safe? J Emerg Med 2011;41:135141.

46. Demaerschalk BM, Durocher DL. How diagnosis-related group 559 will change the US Medicare cost reimbursement ratio for stroke centers. Stroke 2007;38:1309-1312.

47. Demaerschalk BM, Hwang HM, Leung G. Cost analysis review of stroke centers, telestroke, and rt-PA. Am J Manag Care 2010;16:537-544.
48. Lattimore SU, Chalela J, Davis L, et al. NINDS Suburban Hospital Stroke Center. Impact of establishing a primary stroke center at a community hospital on the use of thrombolytic therapy: the NINDS Suburban Hospital Stroke Center experience. Stroke 2003;34:e55-e57.

49. Stroke Treatment and Ongoing Prevention Act (STOP Stroke Act), H.R. 898 and S. 1064, 109th Congress.

50. Gladstone DJ, Rodan LH, Sahlas DJ, et al. A citywide prehospital protocol increases access to stroke thrombolysis in Toronto. Stroke 2009;40:3841-3844. 


\section{Neurology}

\section{Overview of key factors in improving access to acute stroke care Ramy El Khoury, Richard Jung, Ashish Nanda, et al. Neurology 2012;79;S26-S34 \\ DOI 10.1212/WNL.0b013e3182695a2a}

This information is current as of September 24, 2012

Updated Information \& Services

References

Citations

Permissions \& Licensing

Reprints including high resolution figures, can be found at: http://n.neurology.org/content/79/13_Supplement_1/S26.full

This article cites 45 articles, 29 of which you can access for free at: http://n.neurology.org/content/79/13_Supplement_1/S26.full\#ref-list-1

This article has been cited by 1 HighWire-hosted articles: http://n.neurology.org/content/79/13_Supplement_1/S26.full\#\#otherarti cles

Information about reproducing this article in parts (figures,tables) or in its entirety can be found online at:

http://www.neurology.org/about/about_the_journal\#permissions

Information about ordering reprints can be found online:

http://n.neurology.org/subscribers/advertise

Neurology ${ }^{\circledR}$ is the official journal of the American Academy of Neurology. Published continuously since 1951, it is now a weekly with 48 issues per year. Copyright Copyright (? 2012 by AAN Enterprises, Inc.. All rights reserved. Print ISSN: 0028-3878. Online ISSN: 1526-632X.

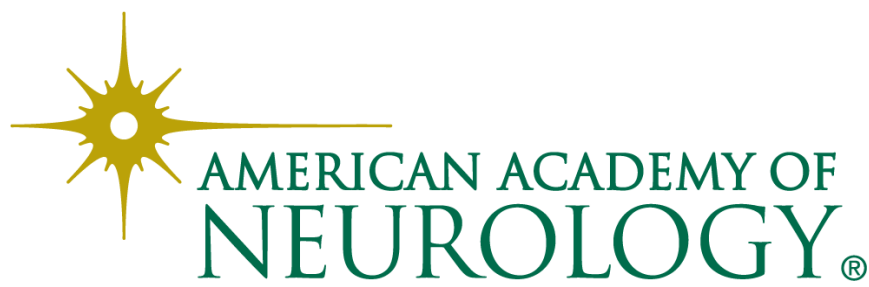

